\title{
Recognition of influenza H3N2 variant virus by human neutralizing antibodies
}

\author{
Sandhya Bangaru, ${ }^{1}$ Travis Nieusma, ${ }^{2}$ Nurgun Kose, ${ }^{3}$ Natalie J. Thornburg,,${ }^{3,4}$ Jessica A. Finn, ${ }^{1}$ \\ Bryan S. Kaplan, ${ }^{5}$ Hannah G. King, ${ }^{3}$ Vidisha Singh, ${ }^{3}$ Rebecca M. Lampley, ${ }^{3}$ Gopal Sapparapu, ${ }^{3,4}$ \\ Alberto Cisneros III, ${ }^{6}$ Kathryn M. Edwards, ${ }^{4}$ James C. Slaughter, ${ }^{3,7}$ Srilatha Edupuganti, ${ }^{8,9}$ Lilin Lai, ${ }^{8,9}$ \\ Juergen A. Richt, ${ }^{10}$ Richard J. Webby, ${ }^{5}$ Andrew B. Ward, ${ }^{2}$ and James E. Crowe Jr. ${ }^{1,3,4}$, \\ 'Department of Pathology, Microbiology and Immunology, Vanderbilt University Medical Center, Nashville, Tennessee, \\ USA. 'Department of Integrative Structural and Computational Biology, The Scripps Research Institute, La Jolla, California, \\ USA. ${ }^{3}$ Vanderbilt Vaccine Center, Vanderbilt University Medical Center, Nashville, Tennessee, USA. ${ }^{4}$ Department of \\ Pediatrics, Vanderbilt University Medical Center, Nashville, Tennessee, USA. IInfectious Diseases, St. Jude Children's \\ Research Hospital, Memphis, Tennessee, USA. ${ }^{6}$ Chemical and Physical Biology Program, Vanderbilt University University, \\ Nashville, Tennessee, USA. 'Department of Biostatistics, Vanderbilt University Medical Center, Nashville, Tennessee, \\ USA. ${ }^{8}$ The Hope Clinic of Emory Vaccine Center, Emory University School of Medicine, Atlanta, Georgia, USA. ${ }^{9}$ Department \\ of Medicine, Division of Infectious Diseases, Emory University School of Medicine, Atlanta, Georgia, USA. ${ }^{10}$ College of \\ Veterinary Medicine, Kansas State University, Manhattan, Kansas, USA.
}

Since 2011, over 300 human cases of infection, especially in exposed children, with the influenza A H3N2 variant (H3N2v) virus that circulates in swine in the US have been reported. The structural and genetic basis for the lack of protection against H3N2v induced by vaccines containing seasonal H3N2 antigens is poorly understood. We isolated 17 human monoclonal antibodies (mAbs) that neutralized H3N2v virus from subjects experimentally immunized with an H3N2v candidate vaccine. Six mAbs exhibited very potent neutralizing activity $\left(\mathrm{IC}_{50}<200 \mathrm{ng} / \mathrm{ml}\right)$ against the $\mathrm{H} 3 \mathrm{~N} 2 \mathrm{v}$ virus but not against current human H3N2 circulating strains. Fine epitope mapping and structural characterization of antigen-antibody complexes revealed that H3N2v specificity was attributable to amino acid polymorphisms in the 150-loop and the 190-helix antigenic sites on the hemagglutinin protein. H3N2v-specific antibodies also neutralized human H3N2 influenza strains naturally circulating between 1995 and 2005. These results reveal a high level of antigenic relatedness between the swine H3N2v virus and previously circulating human strains, consistent with the fact that early human $\mathrm{H} 3$ seasonal strains entered the porcine population in the 1990 s and reentered the human population, where they had not been circulating, as H3N2v about a decade later. The data also explain the increased susceptibility to H3N2v viruses in young children, who lack prior exposure to human seasonal strains from the 1990 s.

Conflict of interest: The authors have declared that no conflict of interest exists.

Submitted: February 23, 2016

Accepted: June 2, 2016

Published: July 7, 2016

Reference information:

JCI Insight. 2016;1(10):e86673.

doi:10.1172/jci.insight.86673.

\section{Introduction}

Annual outbreaks of influenza A viruses (IAVs) in humans are a major global health problem, causing more than 250,000 deaths every year (1). In addition to yearly epidemics, novel influenza viruses originating from other animals periodically cross the species barrier to humans and cause pandemics with high morbidity and mortality rates. IAVs are enveloped viruses that contain the antigenic hemagglutinin (HA) and neuraminidase (NA) surface glycoproteins. HA encodes the receptor-binding site (RBS) and fusion peptide essential for attachment and entry into the host cell and is the primary target for potent neutralizing antibodies (2). The globular head domain that contains the sialic acid-binding (SA-binding) pocket is the major antigenic portion of the HA and tolerates high sequence variability. As a consequence, influenza viruses undergo constant antigenic drift that allows escape from antibody-mediated immunity. There are currently 18 known subtypes of IAVs that fall into 2 broad groups based on the HA sequences and phylogeny (3). Of these, only H1 and H3 subtypes currently circulate in humans. Preferential binding of particular HA molecules to different types of SA receptors on host cells is the major determinant of host specificity (4). The HA of avian IAVs has high affinity for $\alpha$ 2,3-linked SA, whereas human influenza viruses have high affinity for $\alpha 2,6$-linked SA (4-7).

The IAV genome is segmented, and the virus is capable of superinfecting cells with a heterologous IAV 
in a single animal. These features allow for reassortment of the influenza genome in intermediate hosts, such as swine or poultry, enabling emergence of strains that are capable of crossing the species barrier to humans (8). In particular, swine may act as a mixing vehicle for IAVs, because their upper respiratory tract epithelial cells possess both $\alpha$ 2,3- and $\alpha$ 2,6-linked SA receptors, which allow infection with both avian and human IAV (6). Although swine influenza viruses do not generally infect humans, sporadic cases of human infections with swine H1N1 and H3N2 have been documented since 1958 (9). Reassorted swine influenza viruses that are capable of infecting humans can cause severe disease and pose a pandemic threat due to lack of preexisting immunity to the virus. The H1N1 influenza pandemic in 2009-2010 was associated with a virus of swine origins and is an example of a swine virus that was able to transmit easily in the human population and cause disease (10).

Influenza viruses that circulate in pigs are designated "variant" viruses when they cause human infections. Swine-origin IAV H3N2v viruses containing the matrix gene from the $2009 \mathrm{H} 1 \mathrm{~N} 1$ pandemic virus were first detected in humans in July 2011. Since then, there have been at least 345 reported cases of human infections with $\mathrm{H} 3 \mathrm{~N} 2 \mathrm{v}$ viruses, with a high prevalence in children (11-13). A recent study showed that all children $<5$ years old and $>80 \%$ up to 14 years old lack protective serum antibody titers against $\mathrm{H} 3 \mathrm{~N} 2 \mathrm{v}$ (14). Most cases of $\mathrm{H} 3 \mathrm{~N} 2 \mathrm{v}$-associated disease have been associated with exposure to swine, with very limited human-human transmission (12). H3N2v is antigenically distinct from the currently circulating H3N2 seasonal strains, and it has been determined that vaccination with 2010-2011 annual trivalent inactivated virus does not induce neutralizing antibodies against the variant H3N2 virus (14). Lack of preexisting immunity to the variant virus, especially in children, may be a major concern if a highly transmissible H3N2v outbreak occurs (14-16).

Here, we describe the characterization of human mAbs to $\mathrm{H} 3 \mathrm{~N} 2 \mathrm{v} \mathrm{HA}$ isolated from individuals vaccinated with an experimental monovalent inactivated $\mathrm{H} 3 \mathrm{~N} 2 \mathrm{v}$ vaccine candidate. We used these $\mathrm{mAbs}$ to define the molecular basis of strain specificity or cross-reactivity for human neutralizing antibodies recognizing the $\mathrm{HA}$ of $\mathrm{H} 3$ seasonal or emerging $\mathrm{H} 3$ variant viruses. The results indicate that polymorphisms in the 150 helix and the 190 loop, located near the RBS on HA, play a major role in escape of H3N2v virus from immunity induced by seasonal H3N2 vaccines. Furthermore, our results reveal that the HA protein of H3N2v strains is antigenically similar to the human H3N2 IAV strains that circulated during the late 1990s, during which several H3N2 spillover events have been suggested to occur from humans into US swine (17-19).

\section{Results}

Isolation of H3N2v-reactive human mAbs from vaccinated donors. Healthy adult donors received 2 doses of subvirion $\mathrm{H} 3 \mathrm{~N} 2 \mathrm{v}$ vaccine (15 $\mu \mathrm{g}$ of $\mathrm{HA} /$ dose) 21 days apart in an open-label trial, the results of which were reported previously (20). Peripheral blood samples were obtained from volunteers after informed consent on the day of vaccination (day 0) and 21 days after the second dose of vaccine (day 42). Cryopreserved peripheral blood mononuclear cells (PBMCs) were immortalized by EBV transformation, and we collected supernatants from the resulting lymphoblastoid cell lines. Supernatants were screened by ELISA for binding to recombinant HA protein from the H3N2v strain A/Minnesota/11/2010 (designated here as the MNv strain) or one of two representative H3N2 seasonal strains, A/Victoria/361/2011 (designated here as the Victoria strain) or A/Wisconsin/67/2005. The frequency of H3N2 seasonal or variant-reactive $\mathrm{B}$ cell lines was reported previously in the description of the vaccine trial results, and those studies indicated a significant $\mathrm{B}$ cell response to the $\mathrm{H} 3 \mathrm{~N} 2 \mathrm{v}$ HA among the vaccinated individuals (20). The majority of the B cells that secreted H3 HA-reactive antibodies on day 42 recognized the variant HA specifically, with limited cross-reactivity between variant virus-reactive antibodies secreted by B cells and those secreted by seasonal virus-reactive B cells (Supplemental Figure 1; supplemental material available online with this article; doi:10.1172/jci.insight.86673DS1). Transformed B cell lines with supernatants that showed reactivity against the MNv HA in ELISA were selected for fusion to generate human hybridoma cell lines secreting mAbs. We used PBMCs collected on study day 42 from a total of 12 subjects to isolate 36 cloned hybridomas secreting mAbs. The IgG subclass and light chain type of the 17 neutralizing antibodies are presented along with the donor number for the sample from which they were isolated (Figure 1).

Binding and neutralization profile of $H 3 N 2 v$ mAbs. The neutralization potential of the clones in this antibody panel against $\mathrm{MNv}$ virus was determined by microneutralization assay using the MNv virus. Seventeen of thirty-six mAbs exhibited neutralizing activity against the variant virus when tested in concentrations as high as $10 \mu \mathrm{g} / \mathrm{ml}$ (Figure 1). The $\mathrm{IC}_{50}$ values are shown as a heat map, with increased color 


\begin{tabular}{|c|c|c|c|c|c|c|c|c|c|}
\hline \multirow[b]{2}{*}{$\begin{array}{l}\text { Monoclonal } \\
\text { antibody }\end{array}$} & \multirow[b]{2}{*}{$\begin{array}{l}\text { Subject } \\
\text { number }\end{array}$} & \multirow[b]{2}{*}{$\begin{array}{c}\text { IgG } \\
\text { subclass }\end{array}$} & \multirow[b]{2}{*}{ Light chain } & \multicolumn{4}{|c|}{$\mathrm{EC}_{50}(\mathrm{ng} / \mathrm{mL})$ for indicated strain } & \multirow[b]{2}{*}{$\begin{array}{c}\mathrm{H} 3 \mathrm{~N} 2 \mathrm{v} \\
\mathrm{IC} 50 \\
\text { (ng/mL) }\end{array}$} & \multirow[b]{2}{*}{$\begin{array}{c}\mathrm{H} 3 \mathrm{~N} 2 \mathrm{v} \\
\mathrm{HAl} \\
(\mu \mathrm{g} / \mathrm{mL})\end{array}$} \\
\hline & & & & $\begin{array}{c}\text { H3N2v } \\
\text { Minnesota } \\
2010\end{array}$ & $\begin{array}{l}\text { H3N2 } \\
\text { Perth } \\
2009\end{array}$ & $\begin{array}{c}\text { H3N2 } \\
\text { Victoria } \\
2011\end{array}$ & $\begin{array}{c}\text { H3N2 } \\
\text { Texas } \\
2012\end{array}$ & & \\
\hline $\mathrm{H} 3 \mathrm{v}-126$ & 27 & 1 & $\lambda$ & 3 & $>$ & $>$ & $>$ & 3 & 0.16 \\
\hline H3v-71 & 37 & 1 & $\lambda$ & 10 & $>$ & $>$ & $>$ & 7 & 0.31 \\
\hline $\mathrm{H} 3 \mathrm{v}-47$ & 10 & 1 & K & 4 & 18 & 26 & 38 & 8 & $>5.0$ \\
\hline H3v-98 & 37 & 1 & K & 19 & $>$ & $>$ & $>$ & 14 & 0.32 \\
\hline $\mathrm{H} 3 \mathrm{v}-81$ & 37 & 1 & $\lambda$ & 29 & $>$ & $>$ & $>$ & 59 & 1.25 \\
\hline $\mathrm{H} 3 \mathrm{v}-141$ & 54 & 1 & $\mathrm{~K}$ & 25 & $>$ & $>$ & $>$ & 137 & 1.25 \\
\hline $\mathrm{H} 3 \mathrm{v}-45$ & 9 & 3 & $\lambda$ & 14 & $>$ & $>$ & $>$ & 257 & 1.25 \\
\hline H3v-95 & 37 & 3 & $\lambda$ & 9 & 159 & 1 & 239 & 402 & 2.5 \\
\hline H3v-62 & 37 & 1 & $K$ & 4 & $>$ & 1,625 & $>$ & 438 & $>5.0$ \\
\hline $\mathrm{H} 3 \mathrm{v}-86$ & 37 & 1 & $K$ & 27 & 357 & 13 & 368 & 956 & $>5.0$ \\
\hline $\mathrm{H} 3 \mathrm{v}-7$ & 1 & 1 & $K$ & 34 & $>$ & $>$ & $>$ & 1,226 & $>5.0$ \\
\hline $\mathrm{H} 3 \mathrm{v}-9$ & 2 & 1 & $K$ & 38 & $>$ & $>$ & $>$ & 1,301 & $>5.0$ \\
\hline H3v-79 & 37 & 1 & $\lambda$ & 86 & 353 & $>$ & 219 & 1,602 & $>5.0$ \\
\hline
\end{tabular}

Figure 1. Characterization of 17 neutralizing monoclonal antibodies (mAbs). The antibodies are arranged in the order of neutralization potency (column 9) with the most potent antibodies at the top. Seventeen mAbs isolated by human B cell hybridoma generation exhibited neutralization potential (shown as half-maximal inhibitory concentration $\left[\mathrm{IC}_{50}\right]$ ) at $<5 \mu \mathrm{g} / \mathrm{ml}$ against the $\mathrm{H} 3 \mathrm{~N} 2 \mathrm{v}$ virus by microneutralization assay. Nine antibodies exhibited hemagglutinin inhibition (HAI) activity, indicating that they disrupt receptor-binding function of the virus. The mAbs were tested for binding against $\mathrm{HA}$ from $\mathrm{H} 3 \mathrm{~N} 2 \mathrm{~V}$ or 3 seasonal strains (shown as half-maximal effective concentration $\left[\mathrm{EC}_{50}\right]$ ). The $>$ symbol indicates that binding was not detected at the maximum concentration tested $(2 \mu \mathrm{g} / \mathrm{ml})$. The experiments for determining $\mathrm{EC}_{50}(n=4), \mathrm{IC}_{50}(n=3)$, and $\mathrm{HAI}(n=3)$ were conducted twice independently.

intensity corresponding to an increase in neutralizing potency. Six mAbs (found at the top of Figure 1 and designated as $\mathrm{H} 3 \mathrm{v}-126, \mathrm{H} 3 \mathrm{v}-71, \mathrm{H} 3 \mathrm{v}-47, \mathrm{H} 3 \mathrm{v}-104, \mathrm{H} 3 \mathrm{v}-98$, and $\mathrm{H} 3 \mathrm{v}-81$ ) showed very potent neutralization against the virus, with $\mathrm{IC}_{50}$ values of less than $100 \mathrm{ng} / \mathrm{ml}$. We also determined the ability of $4 \mathrm{mAbs}$ (H3v-98, H3v-104, H3v-71, and H3v-45) to neutralize $4 \mathrm{H} 3 \mathrm{~N} 2$ strains representing each antigenic cluster circulating in swine, A/Swine/Texas/4199-2/98 (cluster I), A/Swine/Colorado/23619/99 (cluster II), A/Swine/Oklahoma/18089/99 (cluster III), and A/Ohio/13/2012 (cluster IV) (Supplemental Table 1). $\mathrm{H} 3 \mathrm{v}-98$ and $\mathrm{H} 3 \mathrm{v}-104$ showed potent inhibiting activity against 3 of 4 strains. H3v-71 and H3v-45 exhibited activity against 2 strains at low concentrations.

The $17 \mathrm{H} 3 \mathrm{~N} 2 \mathrm{v}$-neutralizing mAbs were tested for binding to MNv and 3 seasonal strains A/Perth/16/2009, Victoria, and $\mathrm{A} / \mathrm{Texas} / 50 / 2012$. All of the antibodies had half-maximal effective concentrations $\left(\mathrm{EC}_{50}\right)$ for binding below $100 \mathrm{ng} / \mathrm{ml}$ to $\mathrm{MNv} \mathrm{HA}$ (Figure 1). Increasing intensity of the orange cell fill color in the $\mathrm{EC}_{50}$ column corresponds to increasing binding for the indicated HA. The antibodies displayed a differential binding pattern - 9 mAbs bound specifically to the MNv HA, while the other 8 bound to both the variant HA and a combination of seasonal HAs (Figure 1). Interestingly, all but one (H3v-47) of the potently neutralizing mAbs exhibited a variant-specific binding phenotype without detectable cross-reactivity for the HA of H3 seasonal strains (Figure 1) or other HA subtypes (Supplemental Table 2).

We determined the nucleotide sequence of the antibody heavy chain variable gene regions (Table 1). The 17 neutralizing antibodies had unique HCDR3 sequences, indicating that the mAbs represented independent clones. We also performed next-generation sequence analysis of antibody gene repertoires for a subset of donors (donors 10, 27, 37, and 41). Interestingly, we were able to identify on the day of immunization (day 0 ) a member of the clonal lineage for 2 of the $6 \mathrm{mAbs}$ obtained from these donors, suggesting that the clone was induced by prior infection (Supplemental Table 3). The frequency of those clones was greatly expanded on day 7, the expected day of peak plasmablast circulation in peripheral blood (Supplemental Table 3). A clonal lineage of one of the clones, H3v-104, is shown in Supplemental Figure 2, with the amino acid sequence alignment of 296 clonal variants shown in Supplemental Table 4.

Potently neutralizing $m A$ bs block the RBS. Due to the HA specificity demonstrated by the mAbs, we anticipated 
Table 1. Antibody variable genes encoding 17 H3v-reactive human mAbs

\begin{tabular}{|c|c|c|c|c|c|}
\hline \multirow[t]{2}{*}{$\mathbf{m A b}$} & \multicolumn{3}{|c|}{ Heavy chain } & \multirow[t]{2}{*}{ HCDR3 length } & \multirow[t]{2}{*}{ HCDR3 amino acid sequence } \\
\hline & $\mathbf{V}_{\mathrm{H}}$ & D & $J_{H}$ & & \\
\hline H3v-126 & $2-70 D^{*} 04$ & $6-6^{*} 01$ & $4^{*} 02$ & 16 & ARTDGGSISSAAYFES \\
\hline H3v-71 & $1-69 * 01$ & $3-10 * 02$ & $5^{*} 02$ & 16 & AREGLGSVIIGPWFDP \\
\hline H3v-47 & $1-69 * 05$ & $2-2^{*} 01$ & $3 * 02$ & 21 & ARGASKVEPAAPAYSDAFDM \\
\hline H3v-104 & $1-69 * 13$ & $5-12 * 01$ & $4 * 02$ & 16 & ARDYYRGEFSGYDFES \\
\hline H3v-98 & $3-23^{*} 01$ & $1-1 * 01$ & $4 * 02$ & 13 & AKSSFTKGSPFDY \\
\hline H3v-81 & $3-49 * 04$ & $1-7^{*} 01$ & $6^{*} 02$ & 19 & SREAANWNYPYHYSNGMDV \\
\hline $\mathrm{H} 3 \mathrm{v}-141$ & $3-15^{*} 01$ & $3-10 * 01$ & $4^{*} 02$ & 15 & TTDNSFYYGSGYFDH \\
\hline $\mathrm{H} 3 \mathrm{v}-45$ & $3-9 * 01$ & $1-26 * 01$ & $5^{*} 02$ & 17 & AKDGASGGTYYEAGFDP \\
\hline H3v-95 & $3-21^{*} 01$ & $5-18 * 01$ & $3 * 02$ & 15 & ARDLSVYSYGGAFDI \\
\hline H3v-62 & $3-30 * 04$ & $2-15^{*} 01$ & $5^{*} 02$ & 14 & ARRFCTGGSCYLDP \\
\hline H3v-84 & $3-48 * 02$ & $3-16^{*} 01$ & $4^{*} 02$ & 14 & ARDGAVVFGVPFDT \\
\hline H3v-11 & $1-18^{*} 01$ & $2-21^{*} 01$ & $4^{*} 02$ & 17 & ARRSRAWGLSKQQGPLDY \\
\hline H3v-21 & $1-2 * 02$ & $5-18 * 01$ & $4^{*} 02$ & 14 & ARGYNLGYLVLFDY \\
\hline H3v-86 & $6-1^{*} 01$ & $3-22 * 01$ & $4^{*} 02$ & 15 & ARGIQHWWMMVAFDH \\
\hline H3v-7 & $3-33^{*} 04$ & $2-15^{*} 01$ & $4^{*} 02$ & 15 & AKDRDGGVARAPLDY \\
\hline H3v-9 & $4-34^{*} 01$ & $3-3 * 01$ & $4^{*} 02$ & 20 & ARGRPSDESWSGYLDNGFDF \\
\hline H3v-79 & $1-69 * 02$ & $6-19 * 01$ & $6 * 02$ & 22 & AVRAFSTAVAGKGPWHYYGMDV \\
\hline
\end{tabular}

that the majority of the potently neutralizing mAbs mediated neutralization by binding to the less conserved head domain of HA. We performed hemagglutination inhibition (HAI) assays in order to identify mAbs that interfered with the receptor-binding function of HA. A total of 9 of the 17 neutralizing mAbs exhibited HAI activity against the MNv virus, suggesting that these mAbs function by blocking virus binding to the SA receptor (Figure 1). The most potent $\mathrm{mAbs}$ that displayed variant-specific binding phenotype also exhibited HAI activity, suggesting that the variant-specific polymorphisms around the RBS in the head domain region on MNv HA play a major role in determining the unique antigenic profile of the variant virus.

In vivo efficacy of $H 3 v-126, H 3 v-104$, and $H 3 v-71$. We tested representative $\mathrm{mAbs}$ as prophylaxis in a mouse challenge study. Groups of 6- to 8-week-old female DBA/2J mice at 5 animals per group were injected with $100 \mu \mathrm{g}$ of individual $\mathrm{mAbs}$ by the intraperitoneal route on the day prior to virus challenge. Controls $(n=10)$ were injected with PBS. The modestly increased body weight for all 3 groups of mAb-treated animals when compared with the PBS-treated animals was not statistically different (Figure 2).

Competition-binding studies. In order to determine if the neutralizing $\mathrm{mAbs}$ bound to common or diverse epitopes on HA, we performed competition-binding assays using biolayer interferometry with all of the

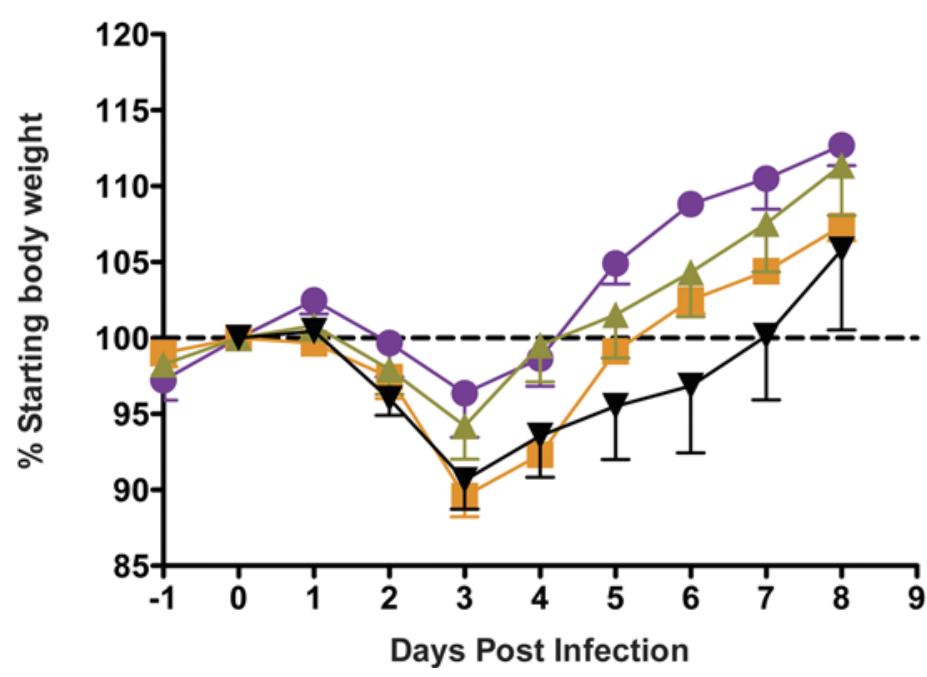

$H 3 v-126$ $\mathrm{H} 3 \mathrm{v}-104$ H3v-71 PBS neutralizing antibodies. Fifteen of the seventeen neutralizing mAbs were classified into competition-binding groups based on their ability to block other mAbs from binding to the HA; we were unable to detect good binding signal for $\mathrm{H} 3 \mathrm{v}-81$ or $\mathrm{H} 3 \mathrm{v}-7$ with biolayer interferometry. The 15 neutralizing mAbs tested fell into 3 major competition-binding groups, with some overlap between the

Figure 2. Prophylactic efficacy of H3v-104, H3v-126, and H3v-71 in mice. Groups of mice $(n=5)$ were treated with $100 \mu \mathrm{g}$ of individual mAbs 24 hours before challenge with mouse-adapted A/Minnesota/11/2010 X203 virus. Controls $(n=10)$ were injected with PBS. The weight loss of mice was measured daily for 14 days after inoculation (day 0 ). 


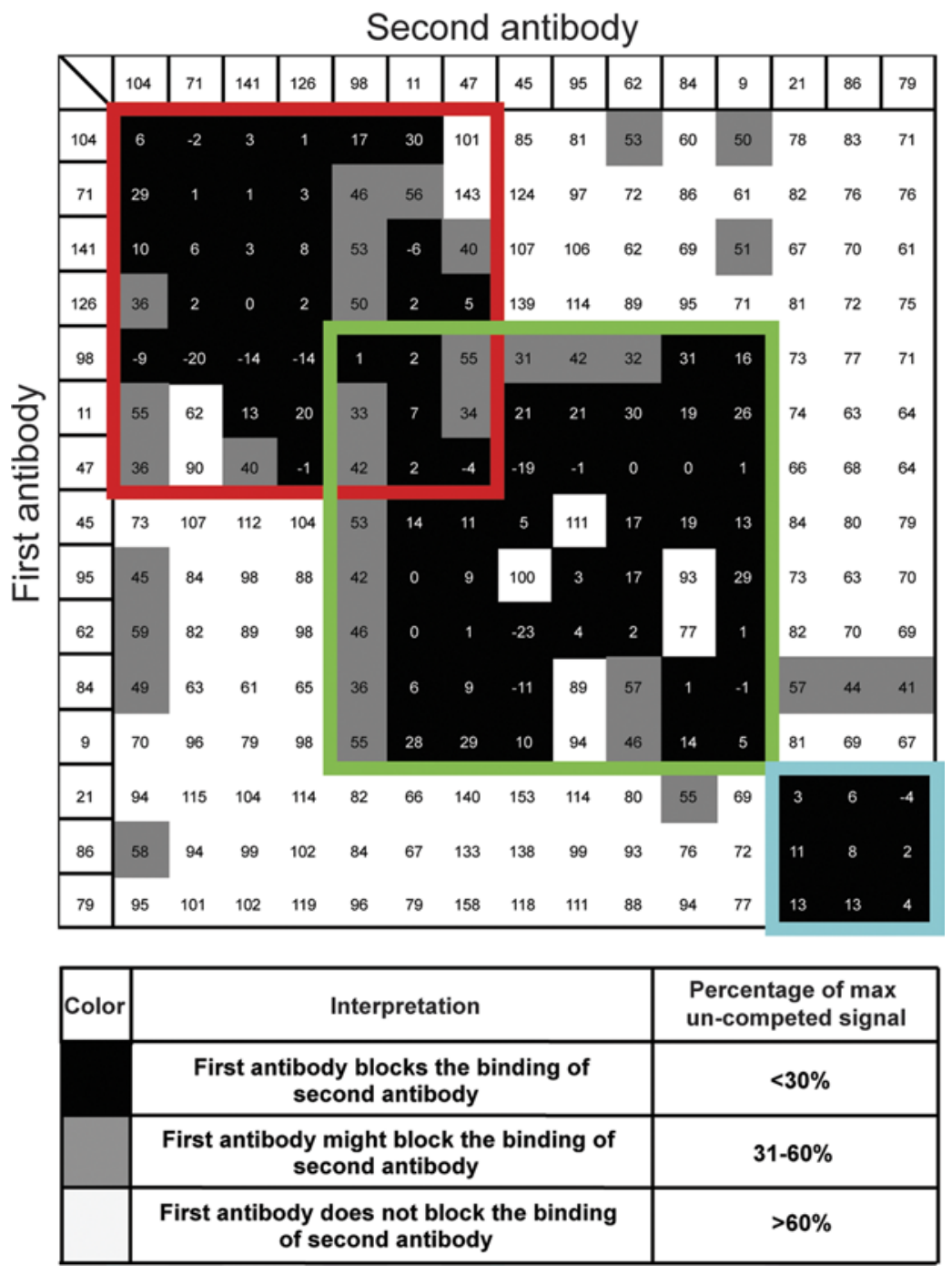

Figure 3. Competition binding of neutralizing antibodies to H3N2v A/Minnesota/11/2010 hemagglutinin (HA) protein. Biolayer interferometry was used to perform competition-binding assays. The HA was loaded onto Ni-NTA tips, and binding to 2 successive antibodies was tested. The binding signal for each antibody was obtained from a single association step of the mAb onto HA. If binding of the first antibody blocked the binding of the second antibody by reducing its binding signal by more than $70 \%$, it was defined as a competitor, indicated in black. The values in the table indicate the percentage of the maximum uncompeted binding signal. The red box indicates group 1 , the binding group comprising the potently neutralizing mAbs. The green box and the blue box represent group 2 (partially overlaps with group 1) and group 3 , respectively. The experiment was conducted twice independently.

groups designated groups 1 and 2 and a distinct group 3 (Figure 3). Notably, 4 of the most potent neutralizing clones (H3v-98, H3v-104, H3v-126, and H3v-71) that also exhibited HAI activity and variant specificity fell into the same competition-binding group, group 1 .

Mutagenesis experiments and electron microscopy revealed regions on the $H 3 N 2 v H A$ important for its immune escape from antibodies induced by seasonal H3N2 viruses. The 6 most potently neutralizing $\mathrm{mAbs}$ that displayed a variant-specific phenotype (i.e., mAbs that bound specifically to the MNv HA and not to the seasonal HAs) were chosen for fine epitope mapping. We sought to determine the residues important for immune escape of the $\mathrm{H} 3 \mathrm{~N} 2 \mathrm{v}$ virus from antibodies induced by seasonal vaccines. We considered that the $\mathrm{HA}$ of $\mathrm{MNv}$ has 52 polymorphisms as compared with the Victoria seasonal strain. We initially performed a mutagenesis screen by introducing variant-specific polymorphisms into a cDNA encoding the Victoria HA protein to identify mutations that would enhance binding to the variant-specific mAbs. 17 variant-specific polymorphisms were introduced into Victoria HA as single or double mutations. Three double-mutant HA molecules, Victoria I202V/T203I, A163E/L164Q, and R142G/N144V, enhanced binding to mAbs H3v-98 and $\mathrm{H} 3 \mathrm{v}-104$ in comparison to wild-type Victoria HA (Supplemental Figure 3).

Based on the results of the initial mutagenesis screen, we targeted the region around the RBS and introduced residues from seasonal strains into the H3N2v HA to identify residues that disrupted binding to wild-type $\mathrm{MNv}$. The $\mathrm{EC}_{50}$ values for $\mathrm{MNv} \mathrm{HA}$ mutants that disrupt binding to each antibody are shown with representative binding curves (Figure 4). Mutation of Y155T/N156K/L157S disrupted binding to both mAb H3v-71 and mAb H3v-81, whereas mutant MNv N158G/Y159S/K160T did not bind to mAb H3v-71 and MNv K189N/T192I/N193R did not bind to H3v-81. A single-mutation Y137S disrupted binding to $\mathrm{H3v}-141$. In addition, a triple alanine mutant, MNv L194A/Y195A/V196A, did not bind to mAbs H3v-98, H3v-104, H3v-71, H3v-81, and H3v-104.

We also performed electron microscopy (EM) of MNv HA in complex with Fab portions of the 3 most potent variant-specific antibodies, $\mathrm{H} 3 \mathrm{v}-104, \mathrm{H} 3 \mathrm{v}-126$, and $\mathrm{H} 3 \mathrm{v}-71$ (Figure 5, A-C), along with the Fab of a stem-binding antibody CR9114 (used as reference in each complex). The EM reconstructions revealed that all 3 antibodies bound to the RBS on HA, with overlapping footprints (Figure 5D). This finding was consistent with the competition-binding data and demonstrated that H3v-126, H3v-71, and H3v-104 all fall under the same competition-binding group (group 1). The H3v-104 mAb displayed a comparatively broader footprint on HA, extending below the RBS, supporting our previous observation that the Victoria R142G/N144V mutant enhanced binding to mAb H3v-104 but not to H3v-126 or H3v-71. H3v-71 bound 
A

\begin{tabular}{|c|c|c|c|c|c|c|c|}
\hline \multirow{2}{*}{$\begin{array}{l}\text { Graph } \\
\text { Legend }\end{array}$} & \multirow{2}{*}{ H3N2v MN mutants } & \multicolumn{6}{|c|}{$E_{50}(\mu \mathrm{g} / \mathrm{mL})$} \\
\hline & & H3v-98 & H3v-104 & H3v-71 & H3v-81 & H3v-141 & H3v-126 \\
\hline$-\triangle$ & MN wild-type & 0.13 & 0.05 & 0.09 & 0.29 & 0.07 & 0.04 \\
\hline 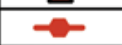 & MN Y137S & 0.13 & 0.06 & 0.09 & 0.34 & $>$ & 0.04 \\
\hline- & MN Y155T/N156K/L157S & 0.19 & 0.06 & $>$ & $>$ & 0.30 & 0.04 \\
\hline 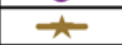 & MN N158G/Y159S/K160T & 0.30 & 0.08 & $>$ & 0.32 & 0.45 & 0.04 \\
\hline-7 & IMN K189N/T192I/N193R & 0.18 & 0.06 & 0.12 & $>$ & 0.61 & 0.04 \\
\hline- & MN L194A/Y195A/V196A & 2.89 & 9.00 & $>$ & $>$ & $>$ & 0.06 \\
\hline
\end{tabular}

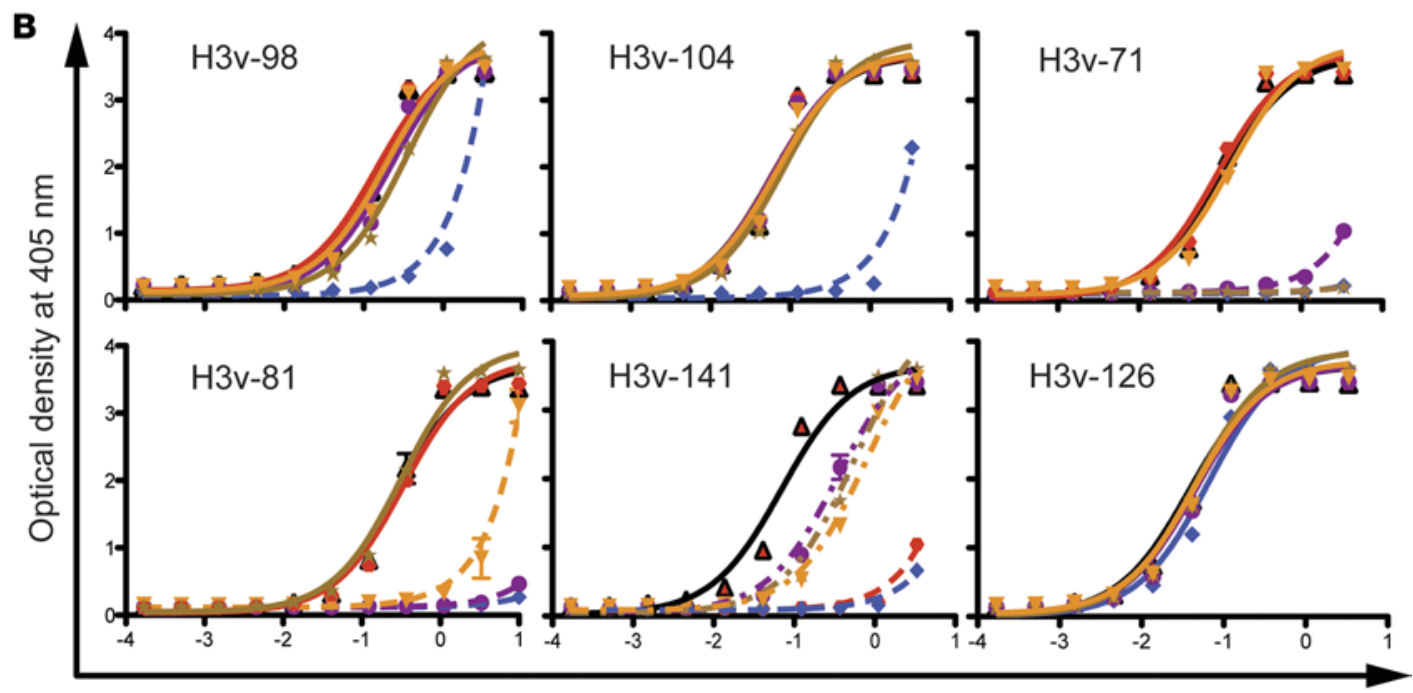

$\log _{10}(\mu \mathrm{g} / \mathrm{mL})$

Figure 4. Binding of H3 variant-specific antibodies to mutated Minnesota hemagglutinin (HA) proteins. Mutagenesis of MNv HA was performed to determine antigenic residues important for recognition by variant-specific mAbs. Mutants of H3N2v A/Minnesota/11/2010 (MN) HA were generated by site-directed mutagenesis, and the half-maximal effective concentration $\left(\mathrm{EC}_{50}\right.$ ) values were determined by performing ELISA with serial dilutions of each antibody against the mutant HAs. The table $(\mathbf{A})$ shows $\mathrm{EC}_{50}$ values and the graph (B) shows binding curves. The mutants that disrupted binding completely or decreased the $\mathrm{EC}_{50}$ by greater than 5 -fold are represented as dashed or dotted lines, respectively, and are indicated by red $\mathrm{EC}_{50}$ values in the table. The $>$ symbol indicates that binding was not detected at the maximum concentration tested $(10 \mu \mathrm{g} / \mathrm{ml})$. The experiments for determining the $\mathrm{EC}_{50}(n=4)$ values were performed twice independently.

similarly to H3v-126 but appeared to interact more with the upper rim of the RBS (Figure 5D). This finding could explain why mutations in the 190 helix and 150 loop affected binding of H3v-71 but not of H3v-126. Collectively, the results from mutagenesis and EM structural studies indicated that variant-specific HA residues residing in 2 major structural features surrounding the RBS, the 150 loop and the 190 helix, principally account for antigenic distinction of the variant virus from current seasonal strains.

The HA of H3N2v virus is antigenically related to that of older human seasonal H3N2 IAVs. The majority of the severe cases of influenza infection caused by H3N2v viruses to date in the US have occurred in children, suggesting partial immunity in adults. We sought to identify the nature of preexisting immunity to the variant virus in the adult population that might provide partial protection against severe disease. Soluble HA proteins belonging to 12 different seasonal H3N2 strains that circulated in humans from 1968 to 2013 were cloned, expressed, and assayed for binding to 6 potently neutralizing mAbs with variant-specific binding and neutralizing phenotypes. Interestingly, $3 \mathrm{mAbs}, \mathrm{H} 3 \mathrm{v}-98, \mathrm{H} 3 \mathrm{v}-104$, and $\mathrm{H} 3 \mathrm{v}-71$, showed strong binding to H3N2 viruses that circulated between 1997 and 2004 but not to the HA of H3 subtype strains that circulated before or after that period (Supplemental Figure 4). To determine if these mAbs had the potential to neutralize these older seasonal strains, we performed microneutralization assays to test activity against 12 viruses that circulated between 1968 and 2013. Indeed, H3v-98 and H3v-104 neutralized all of the strains tested between 1995 and 2005, whereas H3v-71 neutralized all of the viruses between 1997 

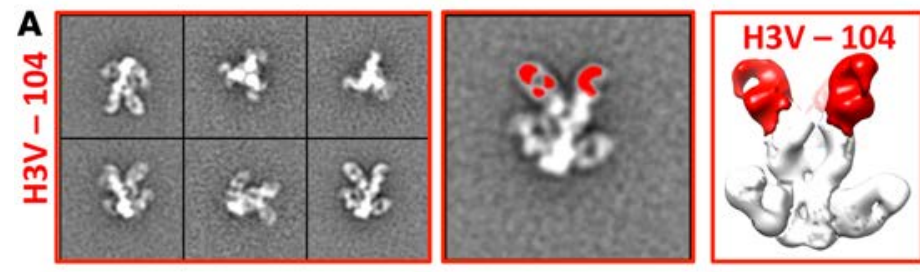

$\mathbf{B}$
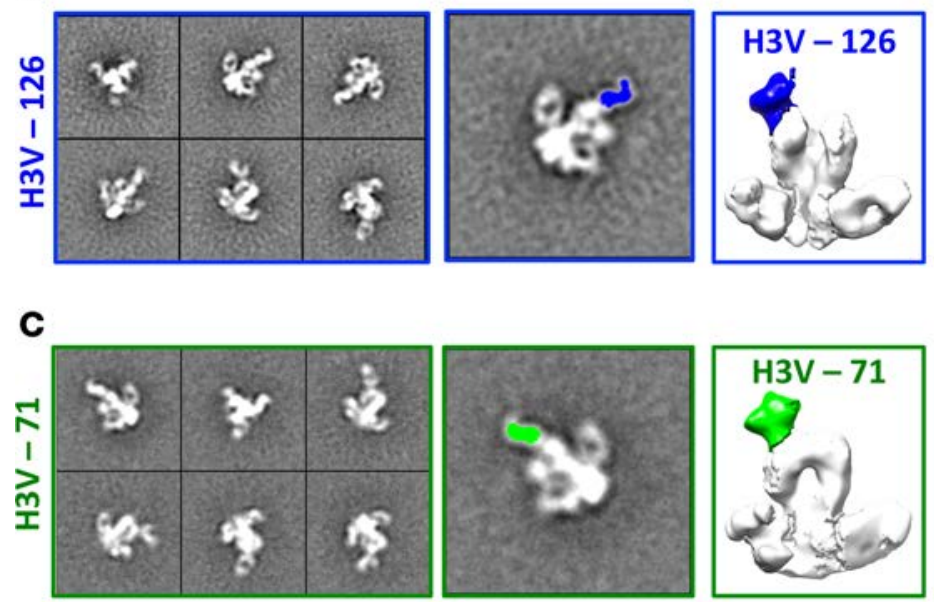
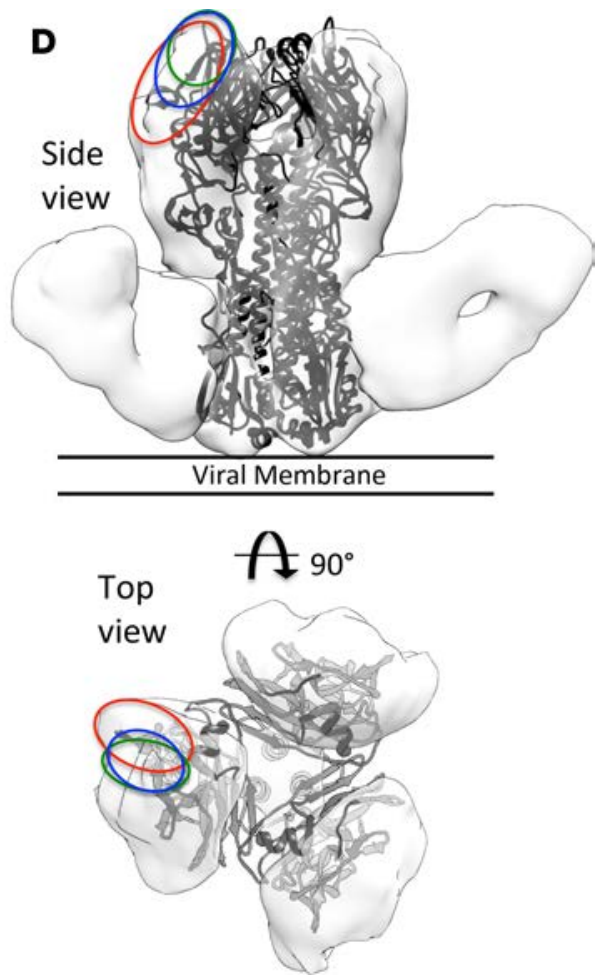

Figure 5. Negative stain EM images of hemagglutinin-Fab complexes. In each case the stem-binding antibody CR9114 was added to the complex in order to improve 3D reconstructions. (A) Reference-free 2D class averages of complex containing Fab 104 (left), single class average with Fab colored in red (middle), and 3D reconstruction (right). (B) Reference-free $2 \mathrm{D}$ class averages of complex containing Fab 126 (left), single class average with Fab colored in blue (middle), and 3D reconstruction (right). (C) Reference-free 2D class averages of complex containing Fab 71 (left), single class average with Fab colored in green (middle), and 3D reconstruction (right). (D) Side and top views of HA-Fab 126-CR9114 with Fab 126 removed and crystal structure of H3V (4FNK) fitted. Binding sites of the 3 antibodies described in A-C is highlighted using colors corresponding to Fabs.

and 2002 (Figure 6). We used a representative panel of live-virus H3 seasonal strains for the neutralization assays that matched as closely as possible the strains that we had used to make recombinant HA molecules for the seasonal strain HA-binding assays. Although we did not detect binding for mAbs H3v-98 or H3v-104 to the HA from A/New York/55/2004 or A/Hiroshima/52/2005, we did observe potent neutralization against the A/Wisconsin/67/2005 strain that was available for neutralization testing. In summary, prior infection with seasonal H3N2 strains that circulated during 2004 and 2005 might induce antibodies that cross-react with $\mathrm{H} 3 \mathrm{~N} 2 \mathrm{v}$ viruses but likely does not guarantee the presence of protective antibodies against $\mathrm{H} 3 \mathrm{~N} 2 \mathrm{v}$ because of antigenic heterogeneity.

\section{Discussion}

We report here the isolation of human mAbs against $\mathrm{H} 3 \mathrm{~N} 2 \mathrm{v}$ virus and the use of them to determine the molecular basis for the antigenic distinction between $\mathrm{H} 3$ seasonal strains and the $\mathrm{H} 3 \mathrm{~N} 2 \mathrm{v}$ viruses. Seventeen antibodies neutralized MNv $\mathrm{H} 3 \mathrm{~N} 2 \mathrm{v}$ at concentrations of less than $10 \mu \mathrm{g} / \mathrm{ml}$. Four of the seventeen neutralizing mAbs exhibited ultrapotent neutralizing activity $\left(\mathrm{IC}_{50}<10 \mathrm{ng} / \mathrm{ml}\right)$. Three of these four ultrapotent neutralizing $\mathrm{H} 3 \mathrm{~N} 2 \mathrm{v}$ mAbs also displayed potent inhibiting activity against swine H3N2 strains belonging to different antigenic clusters. Collectively, these results suggest that some of these H3N2v mAbs could be used in humans as therapeutics against many H3N2 strains circulating in swine in the case of zoonotic transmission event.

The H3 HA-binding breadth of IgG secreted by memory B cells from individuals vaccinated with monovalent inactivated $\mathrm{H} 3 \mathrm{~N} 2 \mathrm{v}$ revealed that there is limited cross-reactivity between antibodies secreted by variant-reactive $B$ cells and $\mathrm{H} 3$ seasonal virus-reactive $B$ cells. About half of the $\mathrm{H} 3 \mathrm{v}$-reactive clones that were isolated displayed specific binding to the MNv HA, with no detectable cross-reactivity for HAs from 3 recent seasonal $\mathrm{H} 3 \mathrm{~N} 2$ strains. The potently neutralizing variant-specific antibodies clustered into the same competition-binding group and used the same virus neutralization mechanism by blocking the RBS on HA. 


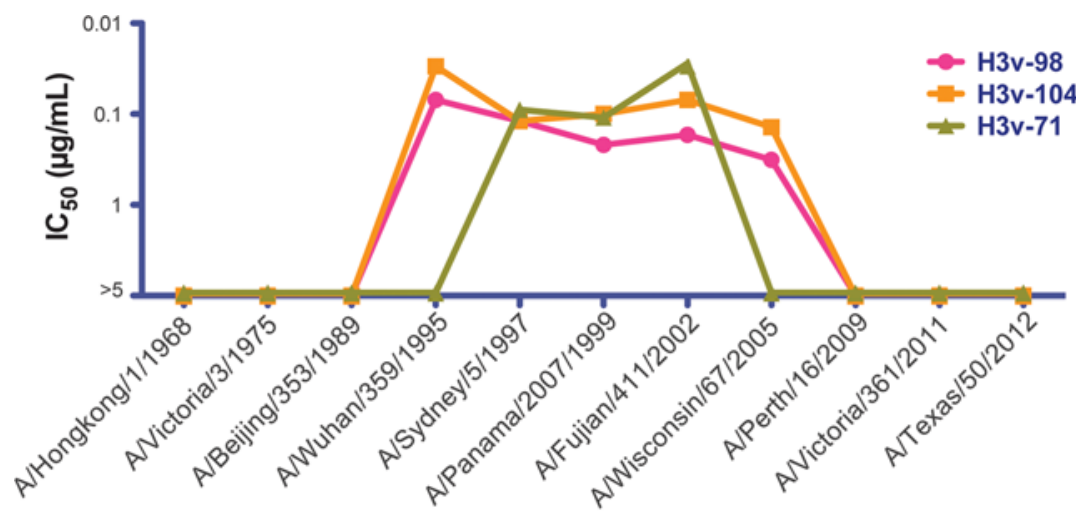

H3N2 seasonal strains from indicated year
Figure 6. Neutralization of H3N2 seasonal strains. The monoclonal antibodies were tested for neutralization activity by microneutralization assay against 11 seasonal strains isolated between 1968 and 2012. The half-maximal inhibitory concentration ( $\mathrm{IC}_{50}$ ) values for $\mathrm{H3V}-98$ (pink), $\mathrm{H3v}-104$ (orange), or $\mathrm{H} 3 \mathrm{v}-71$ (green) are indicated on the $y$ axis, and the H3N2 strains used for neutralization are indicated on the $x$ axis. The $\mathrm{IC}_{50}$ values are represented as baseline if neutralization was not detected at any concentration less than $5 \mu \mathrm{g} / \mathrm{ml}$. The microneutralization assay for determining the $\mathrm{IC}_{50}(n=3)$ values was performed twice independently.

This finding suggested that common antibodies induced by seasonal trivalent inactivated influenza virus (TIV) vaccination that recognize immunodominant epitopes adjacent to the RBS on HA fail to contribute to cross-protection against variant virus. A recent study showed that the molecular basis for antigenic drift in human H3N2 seasonal strains from 1968 to 2006 was attributed to 7 single amino acid substitutions at positions 145, 155, 156, 158, 159, 189, and 193 in HA (21). Six of these residues are located in the antigenic site B (upper rim of RBS). Fine-epitope mapping of 5 potently neutralizing $\mathrm{H} 3 \mathrm{~N} 2 \mathrm{v}$-specific mAbs revealed a similar pattern in context to $\mathrm{H} 3 \mathrm{~N} 2 \mathrm{v}$ antigenicity. The residues important for variant specificity of these mAbs were located primarily in the 150-loop and 190-helix antigenic elements near the RBS.

Historically, swine IAVs have caused sporadic human infections, but these outbreaks had been mostly self-limiting. The 2009 H1N1 pandemic was an example of a swine IAV variant, containing the same subtype of HA and NA as that of the circulating human strain, which caused a human pandemic. A recent rise in swine-origin $\mathrm{H} 3 \mathrm{~N} 2 \mathrm{v}$ human infections has raised concerns of another swine-origin influenza virus pandemic $(11,13)$. Surveillance of IAVs in live-animal markets in Minnesota that included human, swine, and environmental samples provided evidence of interspecies transmission of IAVs from swine to human, signifying a potential risk to persons attending live markets (22). Moreover, H3N2 was identified as the predominant subtype circulating in the swine population; a majority of these swine H3N2 isolates had the same genomic constellation as the $\mathrm{H} 3 \mathrm{~N} 2 \mathrm{v}$ viruses isolated previously from humans (22). Vaccination with seasonal TIV does not provide protection against $\mathrm{H} 3 \mathrm{~N} 2 \mathrm{v}$ viruses, indicating these swine-origin viruses have the potential to spread within unprotected human populations (14).

The HA from the swine-origin 2009 pandemic H1N1 strain is antigenically similar to human H1N1 viruses that circulated between 1918 and 1943 (23). It is hypothesized that IAV classical swine H1N1 was introduced into the domestic swine population sometime during the 1918 human pandemic and remained relatively static in swine for greater than 80 years until the end of the 20th century $(24,25)$. In light of the 2009 pandemic, swine have been thought to act as a potential frozen reservoir of human IAVs, which can result in pandemics through reintroduction of the human-origin virus into a then susceptible human population at a remote time decades later (23). The reactivity of the human mAbs that we isolated from $\mathrm{H} 3 \mathrm{~N} 2 \mathrm{v}$ vaccines revealed that the same phenomenon may be occurring now with $\mathrm{H} 3$ viruses, as human $\mathrm{H} 3$ seasonal strains from the past may be harbored in an antigenically static manner in farm swine in the US.

During late 1990s, there were several spillovers of H3N2 virus from humans to pigs resulting in the introduction of $\mathrm{H} 3 \mathrm{~N} 2$ viruses into the US swine population (17-19). The H3N2v viruses that were isolated from humans in 2011 were phylogenetically closer to seasonal strains that circulated during late 1990s than to current H3 human strains $(19,26)$. We hypothesized that the swine-origin $H 3 N 2 v$ viruses might be antigenically related to the seasonal H3N2 viruses from late 1990s, and thus the H3N2v-specific antibodies that showed no reactivity against the current seasonal strains might recognize the older seasonal viruses. Indeed, we found that 3 potently neutralizing variant-specific mAbs (H3v-98, H3v-104, and H3v-71) had the ability to neutralize seasonal strains that circulated between 1997 and 2002. Additionally, H3v-98 and H3v-104 also neutralized strains from 1995 and 2005. These results indicate that the H3N2v viruses that caused human infections from 2011 to 2013 are antigenically related to the human ancestral H3N2 strains that circulated from 1995 to 2005 . Taken together, the data suggest that children who were born after 2005 
do not have any preexisting cross-reactive immunity against $\mathrm{H} 3 \mathrm{~N} 2 \mathrm{v}$ virus because they were not exposed to the earlier H3 seasonal strains. Therefore, these children should be the priority target population for vaccination to prevent an $\mathrm{H} 3 \mathrm{~N} 2 \mathrm{v}$ outbreak.

The phenomenon of antigenic similarity between swine IAVs and ancestral human strains might be explained by several mechanisms. First, swine may act as a relatively static reservoir of human IAVs. Second, differences in the location of the immunodominant antibody epitopes on HA for swine and humans might allow preservation of antigenic epitopes targeted by humans. We compared the HA1 sequences from H3N2v Minnesota virus and the seasonal Victoria 2011 virus with that of the Sydney 1997 strain (a possible ancestor to the H3N2v Minnesota virus). The HA1 subunit of H3N2v Minnesota and H3N2 Victoria strains has 37 or 32 polymorphisms, respectively, when compared with the HA1 sequence of H3N2 Sydney 1997, suggesting that these strains have drifted at a relatively similar rate in swine compared with the drift in humans. Thirteen of these positions that are situated primarily in the upper or lower rim of the RBS (antigenic sites A and B) showed variation in both Minnesota and Victoria HA. In contrast, there were comparatively more polymorphisms in the H3N2v Minnesota HA sequence in antigenic sites C and E, whereas polymorphisms in the 220-loop (antigenic site D) were seen predominantly in the Victoria 2011 strain. These host-based differences in the observed immunodominance pattern provide a possible explanation of why H3N2 viruses in the swine population retain antigenic features of their human ancestral strains. This recurring phenomenon, in which swine populations act as a static/divergent antigenic reservoir of previously circulating human IAVs should thus be taken into serious consideration for pandemic preparedness.

\section{Methods}

Influenza viruses. The seed stock of $\mathrm{H} 3 \mathrm{~N} 2 \mathrm{v}$ strain $\mathrm{MNv}$ was obtained from Terrence Tumpey (US CDC). The working stocks used for microneutralization assay and HA inhibition assays were made from the supernatant of virus-infected MDCK cell culture monolayers in plain Dulbecco's Modified Eagle Medium (Gibco DMEM, Invitrogen, 11965) with $2 \mu \mathrm{g} / \mathrm{ml}$ of TPCK-trypsin. The seasonal H3N2 strains A/Fujian/411/2002 (FR-1146), A/Perth/16/2009 (FR-370), A/Wisconsin/67/2005 (FR-397), and A/Texas/50/2012 (FR-1210) were provided by the Influenza Reagent Resource (http://www. influenzareagentresource.org/) of the US CDC. Two H3N2 seasonal strains, Victoria (NR-44022) and A/Sydney/5/1997 (NR-12278), were obtained from BEI Resources.

Recombinant soluble $H A$ proteins. Sequences encoding the HA genes of interest were optimized for expression, and cDNAs were synthesized (Genscript) as soluble trimeric constructs by replacing the transmembrane and cytoplasmic domain sequences with cDNAs encoding the GCN4 trimerization domain and a His-tag at the C-terminus. Synthesized genes were subcloned into the pcDNA3.1(+) mammalian expression vector (Invitrogen). HA protein was expressed by transient transfection of $293 \mathrm{~F}$ cells with polyethylenimine transfection reagent and was grown in expression medium (Freestyle 293 Expression Medium; Invitrogen, 12338). The supernatants were harvested after 7 days, filter-sterilized with a $0.4-\mu \mathrm{m}$ filter, and purified with HisTrap TALON FF crude columns (GE Healthcare Life Sciences).

PBMC isolation and hybridoma generation. A cohort of 25 donors was vaccinated twice, 21 days apart, with $15 \mu \mathrm{g}$ of HA/0.5-ml dose of reassortant MNv NYMC X-203, as part of NIH-sponsored clinical research trials of this experimental vaccine (DMID protocol 12-0011). The details of the clinical trial were described previously (20). PBMCs from these donors were isolated from day 0 (day of first vaccination) and protocol day 42 ( 3 weeks after the second dose of vaccine) by density gradient separation on Ficoll and cryopreserved. The human lymphocytes from day 42 were thawed and immortalized by transformation with EBV substrain B95.8 in the presence of CpG10103, cyclosporin A, and a Chk2 inhibitor (27). The cells were plated in a 384-well plate, and 8 days later the supernatants from these transformed B cells were used to screen for the presence of antibodies that bound to soluble H3N2 MNv HA using capture ELISA. The positive wells containing B cells secreting anti-H3N2v antibodies were expanded onto irradiated human PBMC feeder layers for 4 days and then fused with HMMA2.5 myeloma cells using a Cytopulse PA4000 electrofusion device. After fusion, human hybridomas were selected in medium with HAT solution containing ouabain, and several rounds of limiting dilution passages were performed in 384-well culture plates to isolate cell lines of the hybridomas with the highest level of secretion of IgG (27).

$m A b$ production and purification. The hybridoma cell lines with the highest level for IgG expression for each clone were selected as single cells using flow cytometric sorting to obtain clones secreting mAbs. Once hybridoma clones were obtained following sorting and growth in a 384-well plate, we expanded 
them first into wells of a 48 -well plate and then further into a $75-\mathrm{cm}^{2}$ flask to $70 \%$ confluency in hybridoma growth medium (ClonaCell-HY medium E from STEMCELL Technologies, 03805). The cells then were washed and expanded equally to four $225-\mathrm{cm}^{2}$ flasks for antibody expression in serum-free medium (GIBCO Hybridoma-SFM, Invitrogen, 12045084). The supernatant was harvested after 3 weeks, filtered with a $0.4-\mu \mathrm{m}$ filter, and the monoclonal IgGs were purified by affinity chromatography using protein $\mathrm{G}$ columns (GE Life Sciences, Protein G HP Columns).

$E C_{50}$ binding analysis. The $\mathrm{EC}_{50}$ concentration for each antibody was determined as described previously (28). Briefly, we performed ELISA using plates coated with the HA of interest at $2 \mu \mathrm{g} / \mathrm{ml}$ overnight at $4^{\circ} \mathrm{C}$ and then blocked with 5\% nonfat dry milk, 2\% goat serum, and $0.1 \%$ Tween-20 in PBS for 1 hour. Three-fold dilutions of the mAb starting from $10 \mu \mathrm{g} / \mathrm{ml}$ were added to the wells and incubated for 1 hour, followed by incubation for 1 hour at 1:4,000 dilution of anti-human IgG alkaline phosphatase conjugate (Meridian Life Science, W99008A). The plates were washed 3 times between each step with PBS contain ing $0.1 \%$ Tween-20. Phosphatase substrate solution $(1 \mathrm{mg} / \mathrm{ml}$ p-nitrophenol phosphate in $1 \mathrm{M}$ Tris aminomethane) was added to the plates and incubated for 1 hour, and the optical density values were measured at 405-nm wavelength on a BioTek plate reader. Each dilution was done in triplicate, and the $\mathrm{EC}_{50}$ values were calculated in Prism software (GraphPad) using nonlinear regression analysis.

$H A I$ and neutralization assays to determine $I C_{50}$ values. Neutralization potential of all of the mAbs was determined by microneutralization assay and HAI assay. For microneutralization, $50 \mu 1$ of 2 -fold serial dilutions of each antibody starting at $20 \mu \mathrm{g} / \mathrm{ml}$ was incubated with $50 \mu \mathrm{l}$ of $100 \mathrm{TCID}_{50}$ of the virus in viral growth medium (VGM) for 1 hour at room temperature. VGM consists of plain DMEM with $2 \mu \mathrm{g} / \mathrm{ml}$ of TPCK-trypsin and $50 \mu \mathrm{g} / \mathrm{ml}$ gentamicin. The MDCK cell monolayer cultures were washed 2 times with $100 \mu 1$ PBS containing $0.1 \%$ Tween-20, and the virus-antibody mixture then was added to cells and incubated for 32 hours at $37^{\circ} \mathrm{C}$. The cells were washed again and fixed with $100 \mu \mathrm{l}$ of $80 \%$ methanol/20\% PBS. The presence of influenza nucleoprotein in the fixed cells was determined by ELISA using a 1:8,000 dilution of mouse antiNP antibody (BEI Resources, NR 4282) as the primary antibody and a 1:4,000 dilution of goat anti-mouse alkaline phosphate conjugate as the secondary antibody (ThermoFisher Scientific, 31320). Each dilution was tested in duplicate and the half-maximal inhibitory concentration $\left(\mathrm{IC}_{50}\right)$ was determined by nonlinear regression analysis of $\log _{10}$ (inhibitor) vs. response function, using Prism software (GraphPad). An $\mathrm{IC}_{50}$ value of 2 $\mu \mathrm{g} / \mathrm{ml}$ was used as the threshold to determine the presence of functional neutralization. For performing the HAI assay, we used turkey red blood cells (Rockland) that were diluted to $0.5 \%$ in Alsever's solution (Sigma, A3551). $25 \mu$ of 4 hemagglutination units of virus were incubated with $25 \mu 1$ of 2 -fold dilutions of the mAb, starting at $10 \mu \mathrm{g} / \mathrm{ml}$ in PBS for 1 hour at $37^{\circ} \mathrm{C}$. 501 of the virus-antibody mixture was incubated with turkey red blood cells for 1 hour at room temperature. The HAI titer was defined as the highest dilution of antibody that inhibited hemagglutination of red blood cells. Each dilution was performed in duplicate.

In vivo efficacy of H3N2v mAbs. On the day prior to infection, 6- to 8-week-old female DBA/2J mice (Jackson Laboratories, 000671) $(n=5)$ were injected by i.p. route with 1, 10, or $100 \mu$ g of antibody H3v-71, H3v-104, or $\mathrm{H} 3 \mathrm{v}-126$ in $50 \mu \mathrm{PBS}$. Controls were treated with PBS $(n=10)$. On day 0 , mice were inoculated intranasally with $10^{7} \mathrm{PFU}$ of A/Minnesota/10/11 X-203 virus under isoflurane anesthesia and then weighed daily. All treated mice survived for the duration of the study. One animal treated with PBS alone died.

Competition-binding groups. Biolayer interferometry using an Octet Red instrument (ForteBio) was used to confirm mAb-HA binding and to perform competition-binding assays. The HA was loaded onto ForteBio Ni-NTA tips at a concentration of $25 \mu \mathrm{g} / \mathrm{ml}$, and binding to 2 successively applied mAbs at 100 $\mu \mathrm{g} / \mathrm{ml}$ was tested. All of the dilutions were made in $1 \mathrm{X}$ kinetic buffer (ForteBio, 18-5032). The actual binding signal for each $\mathrm{mAb}$ was obtained after 300 seconds of a single association step of the mAb on to HA. If binding of the first antibody blocked the binding of the second antibody by reducing its actual binding signal by more than $70 \%$, it was defined as a competitor. If binding of the first antibody did not block the binding of the second antibody by reducing its actual binding signal by less than $30 \%$, it was defined as a noncompetitor. A signal reduction between $30 \%$ and $70 \%$ was defined as partial blocking.

Site-directed mutagenesis of genes encoding HA proteins. Primers for site-directed mutagenesis were designed using the Agilent QuikChange Primer Design program (Agilent Technologies). The QuikChange Lightning Multi-site Mutagenesis kit (Agilent, 210515-5) was used to introduce multiple mutations into cDNAs encoding the HA genes of H3N2 MNv or H3N2 Victoria, according to the manufacturer's instructions. These mutant HAs were tested for antibody binding in ELISAs, as above, to determine $\mathrm{EC}_{50}$ values for binding and to identify amino acids that comprise the epitope. 
EM. SEC-purified MNv complexed with Fab H3v-104 and Fab CR9114 was diluted to $14.7 \mu \mathrm{g} / \mathrm{ml}$, applied to freshly glow-discharged 400-mesh carbon-coated copper grids, and negatively stained with $2 \%$ uranyl formate. Similar complexes containing MNv and Fab CR9114 were then prepared separately with both Fab H3v-126 and Fab H3v-71 at $7.2 \mu \mathrm{g} / \mathrm{ml}$ and $5.8 \mu \mathrm{g} / \mathrm{ml}$, respectively. The complexes containing Fab H3v-104 or Fab H3v-126 were imaged at $\times 92,000$ magnification on an FEI Talos at $200 \mathrm{keV}$, resulting in a pixel size of $1.57 \AA$ /pixel (calibrated using catalase crystal diffraction), with a dose of $23.96 \mathrm{e} / \AA^{2}$. The complex containing Fab H3v-71 was imaged at $\times 52,000$ magnification on an FEI Tecnai T12 at $120 \mathrm{kV}$ TEM, resulting in a pixel size of $2.05 \AA$ /pixel, with a dose of $25.41 \mathrm{e} / \AA^{2}$. All data were collected using Leginon Multi-Scale Imaging (MSI-raster 3.1) software (29). The Talos is equipped with an FEI Ceta $4 \mathrm{k} \times$ $4 \mathrm{k}$ CMOS and the T12 TEM is equipped with a Teitz F416 $4 \mathrm{k} \times 4 \mathrm{k}$ CMOS.

EM data processing. For the complex containing Fab H3v-104 DoGpicker was used to automatically select particles from 450 raw micrographs that were then binned by 2 , resulting in a $3.14 \AA$ /pixel size, and placed into $128 \times 128$ pixel boxes (30). Particles were aligned with Iterative MRA-MSA and ISAC, resulting in a final stack of 3,618 raw particles $(31,32)$. Class averages from ISAC were used to create a common lines initial model in EMAN2 (33). Model refinement was conducted in EMAN, resulting in a 21.3 A resolution reconstruction based on a 0.5 FSC cutoff value (34). The same processing pipeline was used to prepare a reconstruction of the complex containing Fab H3v-126 from 306 raw micrographs and a final stack of 3,202 particles. Because of variable occupancy of the Fab several rounds of MRA-MSA were conducted to isolate particles containing a single-bound Fab, H3v-126. A model of HA bound with Fab CR9114 only was used as an initial model for refinement, resulting in a resolution of $19.8 \AA$. The complex containing Fab H3v-71 also had variable Fab occupancy. Five rounds of MRA-MSA and one round of ISAC were conducted to isolate particles with only $1 \mathrm{Fab} \mathrm{H3v-71} \mathrm{bound,} \mathrm{resulting} \mathrm{in} \mathrm{a} \mathrm{final} \mathrm{stack} \mathrm{of} \mathrm{3,931}$ particles in 192 pixel boxes. A model of HA bound with Fab CR9114 only was used as an initial model for refinement, resulting in a resolution of $27.2 \AA$.

Antibody heavy and light chain variable gene sequence analysis. Antibody heavy and light chain genes for each of the neutralizing mAbs were cloned from the hybridoma lines after single-cell flow cytometric sorting to biologically clone the cell lines. RNA was extracted from these hybridoma clones using the RNeasy mini kit (Qiagen, 74106), followed by RT-PCR amplification of antibody gene cDNAs. PCR products encoding antibody heavy or light chain genes were cloned individually into the pGEM-T vector, and Sanger nucleotide sequence analysis was used to determine the antibody cDNA sequences. Analysis of variable gene sequences was performed using the international ImMunoGeneTics (IMGT) information system (http://imgt.org/). We also performed next-generation sequence analysis of antibody gene repertoires from 4 selected donors and defined in detail clonal lineage for 1 clone. First, antibody heavy chain variable gene sequences were obtained using RT-PCR and Illumina MiSeq $2 \times 300$ paired-end amplicon sequence analysis from PBMCs collected on day 0, 7, or 42 after immunization. For donor 41 and the H3v-104 clone, we identified those sequences sharing use of the $\mathrm{V}_{\mathrm{H}} 1-69$ and $\mathrm{J}_{\mathrm{H}} 4$ gene segments that encode $\mathrm{H} 3 \mathrm{v}-104$; the complete repertoire sequence database of donor 41 is available at the NCBI Sequence Read Archive at accession SRP075907. Then, CD-HIT was used to identify the HCDR3 sequences of 296 sequences that clustered within $85 \%$ identity with the HCDR3 sequence of $\mathrm{H} 3 \mathrm{v}-104$. We built a Phylip lineage using the alakazam and shazam packages of the Change-O software suite and visualized the network in Cytoscape.

Statistics. The $\mathrm{IC}_{50}$ values were calculated after $\log$ transformation of antibody concentrations using a 3-parameter nonlinear fit analysis of antibody $\log _{10}$ concentration vs. response with $\mathrm{R}^{2}$ values $>0.85$. The $\mathrm{EC}_{50}$ values were calculated after log transformation of antibody concentrations using sigmoidal dose-response nonlinear fit analysis with $\mathrm{R}^{2}$ values of $>0.85$. Change in mouse weights over days $1-8$ were compared by antibody using repeated-measures analysis of covariance, controlling for weight at day 0 . All statistics were analyzed using Prism software version 5 (GraphPad). A $P$ value less than 0.05 was considered significant.

Study approval. PBMCs were collected at the Emory University Vaccine Treatment and Evaluation Unit after informed consent from otherwise healthy subjects with prior history of experimental H3N2v subunit vaccination, as described in the Methods. The protocol and consent form were approved prior to study by the Emory University Institutional Review Board Committee, Atlanta, Georgia, USA. The animal protocol covering the $\mathrm{H} 3 \mathrm{~N} 2 \mathrm{v}$ influenza virus challenge infections of passively immunized mice was reviewed and approved by the Institutional Animal Care and Use Committee at St. Jude Children's Research Hospital. 


\section{Author contributions}

SB, TN, JAF, NJT, BSK, AC, JAR, RJW, ABW, and JEC designed the research study and experiments. SB, TN, JAF, NK, NJT, BSK, HGK, VS, RML, GS, AC, JAR, and ABW conducted laboratory experiments and acquired and analyzed data. BSK and RJW conducted animal studies. JCS gave statistical review. SE, $\mathrm{LL}$, and KME conducted the parental vaccine study and acquired PBMCs. SB and JEC wrote the manuscript. All authors read and commented on the manuscript.

\section{Acknowledgments}

The authors thank F. Smith-House (Vanderbilt) and H. Liu (Kansas State) for technical support, Mark J. Mulligan (Emory) for clinical support during human sample acquisition, and Jill Janssen of the Vanderbilt Clinical Trials Center for regulatory support. The original clinical trial of the H3N2v vaccine (DMID protocol 12-0011) was supported by NIAID, NIH and performed in the Emory Vaccine Center. SB was supported by the Vanderbilt International Scholar Program. This work was supported by NIH grants R01 AI106002 and U19 AI117905; NIAID contracts HHSN272200900047C and HHSN272201400007C (to JEC), HHSN27220800007C (KME), and HHSN272200800005C and HHSN266200700006C (to BSK, RJW, and JAR); and the Vanderbilt NIH Clinical and Translational Science Award UL1 RR024975. The content is solely the responsibility of the authors and does not necessarily represent the official views of the NIH.

Address correspondence to: James E. Crowe Jr., Vanderbilt Vaccine Center, Vanderbilt University Medical Center, 11475 MRB IV, 2213 Garland Avenue, Nashville, Tennessee 37232-0417, USA. Phone: 615.343.8064; E-mail address: james.crowe@vanderbilt.edu.

1. World Health Organization. Influenza (Seasonal). WHO Web site. http://www.who.int/mediacentre/factsheets/fs211/en/ Accessed June 13, 2016.

2. Dreyfus C, et al. Structure of a classical broadly neutralizing stem antibody in complex with a pandemic $\mathrm{H} 2$ influenza virus hemagglutinin. J Virol. 2013;87(12):7149-7154.

3. Tong S, et al. New world bats harbor diverse influenza A viruses. PLoS Pathog. 2013;9(10):e1003657.

4. Suzuki Y, et al. Sialic acid species as a determinant of the host range of influenza A viruses. J Virol. 2000;74(24):11825-11831.

5. Rogers GN, Paulson JC. Receptor determinants of human and animal influenza virus isolates: Differences in receptor specificity of the H3 hemagglutinin based on species of origin. Virology. 1983;127(2):361-373.

6. Ito T, et al. Molecular basis for the generation in pigs of influenza A viruses with pandemic potential. J Virol. 1998;72(9):73677373 .

7. Connor RJ, et al. Receptor specificity in human, avian, and equine H2 and H3 influenza virus isolates. Virology. 1994;205(1):1723.

8. Brown IH. The pig as an intermediate host for influenza A viruses between birds and humans. Int Congr Ser. 2001;1219:173-178.

9. Myers KP, et al. Cases of swine influenza in humans: a review of the literature. Clin Infect Dis. 2007;44(8):1084-1088.

10. Smith GJD, et al. Origins and evolutionary genomics of the 2009 swine-origin H1N1 influenza A epidemic. Nature. 2009;459(7250):1122-1125.

11. Centers for Disease Control Prevention. Update: Influenza A (H3N2)v transmission guidelines — five states, 2011. MMWR Morb Mortal Wkly Rep. 2012;60(51-52):1741-1744.

12. Finelli L, Swerdlow DL. The emergence of influenza A $(\mathrm{H} 3 \mathrm{~N} 2) \mathrm{v}$ virus: what we learned from the first wave. Clin Infect Dis. 2013;57(suppl 1):S1-S3.

13. Lindstrom S, et al. Human infections with novel reassortant influenza A(H3N2)v viruses, United States, 2011. Emerging Infect Dis. 2012;18(5):834-837.

14. Skowronski DM, et al. Cross-reactive and vaccine-induced antibody to an emerging swine-origin variant of influenza $\mathrm{A}$ virus subtype H3N2 (H3N2v). J Infect Dis. 2012;206(12):1852-1861.

15. Centers for Disease Control Prevention. Antibodies cross-reactive to influenza A (H3N2) variant virus impact of 2010-11 seasonal influenza vaccine on cross-reactive antibodies-United States. MMWR Morb Mortal Wkly Rep. 2012;61(14):237-241.

16. Waalen K, et al. Age-dependent prevalence of antibodies cross-reactive to the influenza A (H3N2) variant virus in sera collected in Norway in 2011. Euro Surveill. 2012;17(19): 20170.

17. Zhou NN, et al. Emergence of H3N2 reassortant influenza A viruses in North American pigs. Vet Microbiol. 2000;74(1-2):4758.

18. Zhou NN, et al. Genetic reassortment of avian, swine, and human influenza A viruses in American pigs. $J$ Virol. 1999;73(10):8851-8856.

19. Webby RJ, et al. Evolution of swine H3N2 influenza viruses in the United States. J Virol. 2000;74(18):8243-8251.

20. Keitel WA, et al. Safety and immunogenicity of a subvirion monovalent unadjuvanted inactivated influenza A/H3N2 variant $(\mathrm{H} 3 \mathrm{~N} 2 \mathrm{v})$ vaccine in healthy persons $\geq 18$ years old. J Infect Dis. 2015;212(4):552-561.

21. Koel BF, et al. Substitutions near the receptor binding site determine major antigenic change during influenza virus evolution. Science. 2013;342(6161):976-979.

22. Choi MJ, et al. Live animal markets in Minnesota: A potential source for emergence of novel influenza A viruses and interspecies Transmission. Clin Infect Dis. 2015;61(9):1355-1362. 
23. Manicassamy B, et al. Protection of mice against lethal challenge with $2009 \mathrm{H} 1 \mathrm{~N} 1$ influenza A virus by 1918 -like and classical swine H1N1 based vaccines. PLoS Pathog. 2010;6(1):e1000745.

24. Nelson MI, et al. Multiple reassortment events in the evolutionary history of H1N1 influenza A virus since 1918. PLoS Pathog. 2008;4(2):e1000012.

25. Vincent AL, Ma W, Lager KM, Janke BH, Richt JA. Swine influenza viruses a North American perspective. Adv Virus Res. 2008;72:127-154.

26. Lina B, et al. S-OtrH3N2 viruses: use of sequence data for description of the molecular characteristics of the viruses and their relatedness to previously circulating H3N2 human viruses. Euro Surveill. 2011;16(50):20039.

27. Smith SA, et al. Persistence of circulating memory B cell clones with potential for dengue virus disease enhancement for decades following infection. J Virol. 2012;86(5):2665-2675.

28. Thornburg NJ, et al. Human antibodies that neutralize respiratory droplet transmissible H5N1 influenza viruses. J Clin Invest. 2013;123(10):4405-4409.

29. Suloway C, et al. Automated molecular microscopy: The new Leginon system. J Struct Biol. 2005;151(1):41-60.

30. Voss NR, et al. DoG Picker and TiltPicker: Software tools to facilitate particle selection in single particle electron microscopy. J Struct Biol. 2009;166(2):205-213.

31. Hohn M, et al. SPARX, a new environment for Cryo-EM image processing. J Struct Biol. 2007;157(1):47-55.

32. van Heel M, et al. A new generation of the IMAGIC image processing system. J Struct Biol. 1996;116(1):17-24.

33. Tang G, et al. EMAN2: An extensible image processing suite for electron microscopy. J Struct Biol. 2007;157(1):38-46.

34. Ludtke SJ, et al. EMAN: Semiautomated software for high-resolution single-particle reconstructions. J Struct Biol. 1999;128(1):82-97. 